\title{
Initial Strain Problems Formulation of Combined Materials
}

\author{
Mohamed Abdalla Almherigh \\ Ph. D, M. Eng., B. Sc. Eng. Department of civil Engineering Faculty of Engineering - Tripoli University $P O$ \\ Box 82677, Tripoli, Libya
}

\begin{abstract}
A typical in plane elastic analysis of two combined materials is proposed, using simple and believed to be efficient model that can easily take into consideration, self-weight of used materials, strains due to causes other than loading; such as moisture, temperature, creep and shrinkage are easily incorporated. Simple finite element method formulation is introduced; the two proposed elements for use are presented, then a simple efficient computer program is presented, a list of dominant used references for finite element technique are lastly proposed.
\end{abstract}

Keywords: modeling of mutable materials, finite element modeling of two materials,In-plane stress analysis of reinforced material

\section{Introduction}

The finite element method is a generally applicable technique for getting numerical solutions for almost any problem of stress analysis no matter how complicated is it. Nevertheless, the choice of the different formulations is not a straight forward procedure. Analysis results and accuracy are widely affected not only by how correct are material properties to be used but also by the model(s) and element(s) employed in the program, need not to say that computer programing knowledge is extremely essential.

The use of the Finite Element Method of analysis allows more realistic modeling of material behavior. Depending upon the degree of accuracy required; body forces, Poisson's ratio effects, non-linear behavior and anisotropic material characteristics can all be taken into account. These, of course, are subjected to suitable experimental evaluation of the critical parameters.

\section{Theoritical Formulationofthe Method}

In order to make it possible for the finite element method to solve for strains $\{\varepsilon\}$ that are not due to applied loads, such as thermal strains, creep and shrinkage, we will solve the problem without reference to particular element, thus arriving at a general solution that could be applied to any finite element. While going through this process we will add another condition and that is how we will make provision for strains which are not the result of stress. If an element, irrespective of its shape, degree of freedom or number of components in its stress and strain vectors, has a strain which is not related to load, that strain would not exist if there were no constraints on the element and it could freely change size (as a result of temperature change or other reasons), then this strain will cause certain displacements which might be significant and should not be ignored. When the force $\{\mathrm{f}\}$ is applied, additional displacements will occur giving a total displacement represented by a displacement function $\{\mathrm{u}\}$. Total strains can be found from the displacement function by performing certain differential operations given in the operator matrix $[\Delta]$, that is:

$$
\{\varepsilon\}=\{\Delta\}\{\mathrm{u}\}
$$

But elastic strain is given by; $\varepsilon-\varepsilon_{0}$ and hence the stress is:

$$
\{\sigma\}=[\mathrm{D}]\left\{\varepsilon-\varepsilon_{0}\right\} \quad \text { ii }
$$

Where [D] is the elastic property matrix

The displacement functions $\{\mathrm{u}\}$ are polynomials and can be written as:

$\{\mathrm{u}\}=[\mathrm{P}]\{\rho\} \quad$ iii

Where $[\mathrm{P}]$ contains polynomial terms and $\{\rho\}$ contains the coefficients of the polynomial function. Since the displacement function must give the displacements at the nodes, $\delta$, then when the coordinates of the nodes are substituted into $[\mathrm{P}]$ we get;

$$
\{\delta\}=[\mathrm{A}]\{\rho\} \quad \text { iv }
$$

Where $[\mathrm{A}]$, comes from $[\mathrm{P}]$ evaluated at the nodes. Solving,iv, gives the coefficients in the polynomial:

$\{\rho\}=\left[\mathrm{A}^{-1}\right]\{\delta\}$

Substituting this into, iii, and then into, $\mathrm{i}$, gives;

$\{\varepsilon\}=[\Delta][\mathrm{P}]\left[\mathrm{A}^{-1}\right]\{\delta\} \mathrm{v}$

The operations of $[\Delta]$ can be performed on $[\mathrm{P}]$ giving a new matrix $[\mathrm{B}]$ :

$[\mathrm{B}]=[\Delta][\mathrm{P}] \quad$ vi

Putting, vi, into, v, gives; 
$\{\varepsilon\}=[\mathrm{B}]\left[\mathrm{A}^{-1}\right]\{\delta\} \quad$ vii

Which if substituted into, ii, gives;

$\{\sigma\}=[\mathrm{D}][\mathrm{B}]\left[\mathrm{A}^{-1}\right]\{\delta\}-[\mathrm{D}]\left\{\varepsilon_{0}\right\} \quad$ viii

Now we have an element that has its nodes displaced by $\delta$ and is held in this position by some, as yet unknown force $\{f\}$. From this state let us give the element a vertical displacement $\delta$ which will cause additional strain $\varepsilon *$ within the element, from vii, we get:

$\varepsilon^{*}=\mathrm{BA}^{-1} \delta^{*}$ ix

The additional strain energy stored by the element during this step in an incremental volume is $\varepsilon^{* \mathrm{~T}} \sigma \mathrm{dv}$, which gives for the whole element, by substituting from equations, iiiv, ix:

Increase in strain energy $=\int_{\text {vol }}\left[\mathrm{B} \mathrm{A}^{-1} \delta^{*}\right]^{\mathrm{T}}\left[\mathrm{D} \mathrm{B} \mathrm{A}^{-1} \delta-\mathrm{D} \varepsilon_{0}\right] \mathrm{dv}$

$=\int_{\mathrm{vol}}\left[\delta^{* \mathrm{~T}}\left[\mathrm{~A}^{-1}\right]^{\mathrm{T}} \mathrm{B}^{\mathrm{T}}\right]\left[\mathrm{DB} \mathrm{A}^{-1} \delta-\mathrm{D} \varepsilon_{0}\right] \mathrm{dv} \mathrm{x}$

When the vertical displacement is imposed, the external forces do an amount of work given by:

External work $=\left\{\varepsilon^{*}\right\}^{\mathrm{T}}\{\mathrm{f}\}$

xi

Equating internal and external work done and arranging:

$\{\mathrm{f}\}=\left[\mathrm{A}^{-1}\right]^{\mathrm{T}} \int_{\mathrm{vol}}[\mathrm{B}]^{\mathrm{T}}[\mathrm{D}][\mathrm{B}] \mathrm{dv}[\mathrm{A}]^{-1}\{\delta\}-\left[\mathrm{A}^{-1}\right]^{\mathrm{T}} \int_{\text {vol }}[\mathrm{B}]^{\mathrm{T}}[\mathrm{D}]\left\{\varepsilon_{0}\right\} \mathrm{dv}$ xii

Now if we let: $[\mathrm{K}]=\left[\mathrm{A}^{-1}\right]^{\mathrm{T}} \int_{\mathrm{vol}}[\mathrm{B}]^{\mathrm{T}}[\mathrm{D}][\mathrm{B}] \mathrm{dv}\left[\mathrm{A}^{-1}\right] \quad$ xiii

Then: $\{\mathrm{f}\}=[\mathrm{K}]\{\delta\}-\left[\mathrm{A}^{-1}\right]^{\mathrm{T}} \int_{\text {vol }}[\mathrm{B}]^{\mathrm{T}}[\mathrm{D}]\left\{\varepsilon_{0}\right\}$ dvixx

Equation, ixx relates forces to displacement through $[\mathrm{K}]$ which is the stiffness matrix usually referredtoin its global banded form as [S] and is determined by equation; xiii, and if we let:

$$
\left\{f_{0}\right\}=\left[A^{-1}\right]^{T} \int{ }_{\text {vol }}[B]^{\mathrm{T}}[D]\left\{\varepsilon_{0}\right\} d v
$$

XX

Then equation, ixx, can be rewritten as:

$$
\{\mathrm{f}\}+\left\{\mathrm{f}_{0}\right\}=[\mathrm{K}]\{\delta\} \quad \mathrm{xxi}
$$

In this expression, (eq. xxi) the components of $\{\mathrm{f}\}$ are the actual loads that are imposed on the element and $\left\{\mathrm{f}_{0}\right\}$ components are fictitious forces being in fact the nodal forces that would produce the strain $\left\{\varepsilon_{0}\right\}$, but these not need to be recognized.

To solve a problem we merely calculate $\left\{\mathrm{f}_{0}\right\}$ for each element, given $\varepsilon_{0}$, treat it as though it is a real load and add its components to the proper components of the force vector $\{f\}$. This can be conveniently done while the stiffness matrix is being generated. After all displacements for the assembly have been determined, those that apply to each element are selected in turn and the stresses are found by substituting into (eq. ii).

\section{Elementsproposedinmodeling}

Having developed the theoretical formulation of the stiffness matrixfor any finite element, the next step is to choose the element(s) to be employed in the analysis. The cost of any finite element analysis depends on many factors, among which the type of element chosen in the analysis is one factor.The type of element implies the number of elements, the number of degree of freedom, and the bandwidth to be employed.

The constant strain triangular element (CST) is a simple element with three corner nodes and with two degrees of freedom at each node. The two degrees of freedom are two displacements perpendicular to each other as in the figure next to this section. Because of its simplicity, a CST element does not give good results unless its size is reduced in areas where rapid change of stress is expected. Reducing the size of element, results in a larger number of data points to be plotted and hence in better curve fitting.

The triangular shape of the CST element enables one to break the mesh easily into smaller elements. Moreover, the CST elements do not need a large bandwidth in the stiffness matrix as compared to, for example, linear strain triangle (LST), elements. The usage of the LST elements may roughly double the bandwidth which would mean doubling the space required to store the stiffness matrix in the computer memory.

If the problem geometry is simple, the rectangular element (R E) would be as good as a triangular element.

The bar element as shown later after this sectionhas two degrees of freedom at each end, the two degrees of freedom are two displacements perpendicular to each other. The bar element is bitter to model steel reinforcement and can be used also to model any increase in stiffness i.e lintel. The bar element is extremely simple and it can satisfactorily represent reinforcing steel bars in reinforced concrete for example. Moreover, the bar element also guarantee the compatibility of the displacement degrees of freedom at the common points with CST elements.

Bothe constant strain triangle, and bar element are very will investigated in finite element literature. Hence both elements are not developed here; combining the two elements is believed to be very useful for analyzing cases such as problems of two combined materials as in reinforced masonry or concrete units. 


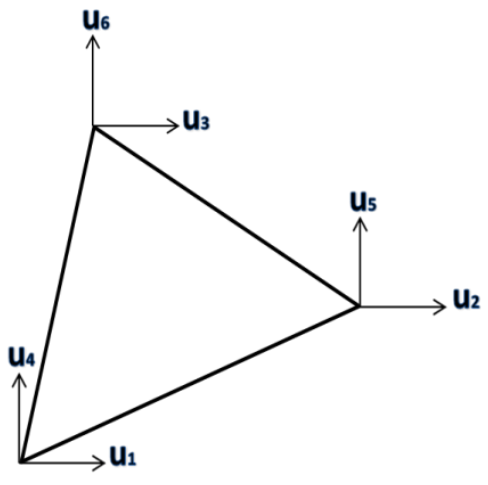

Constant strain triangle element (CST)

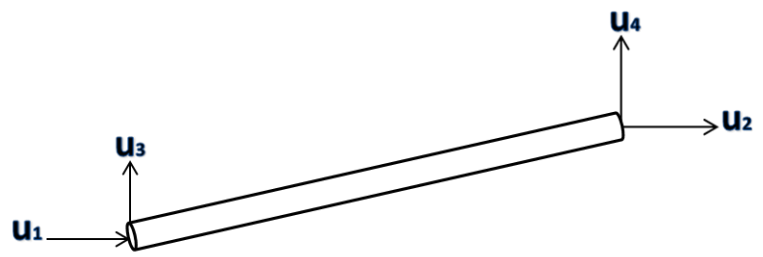

Bar element

\section{Computer Program To Solvefor Thermal Strains}

The work presented in the previous two sections is incorporated in a structural finite element analysis program. Subroutines needed for this program can be readily taken from the widely available literature such as BOWES and RUSSEL, [1], CLAUS-JURGEN BATH, [2], ROBERT D. COOK [3], O. C. ZIENKIEWICZ, [4], I. M. SMITH and D. V. GRIFFITHS [5].

As presented in the flowchart 'next to this section', the program follows the steps mentioned in 'Section 2' of this paper by finding stiffness of each element for both the triangular elements (main material)and the bar element, 'reinforcement elements', and adds the elements stiffness matrix to the global stiffness matrix $[S]$ having dimensions of bandwidth limit or maximum bandwidth times number of degrees of freedom.

Similarly the program builds the force vector $\{\mathrm{F}\}$ by adding the thermal force vector for each element of both materials (basic and reinforcement) to the global force vector $\{F\}$. The program also takes both materials selfweight into consideration.

When all stiffness and forces corresponding to each element have been incorporated into $[\mathrm{S}]$ and $\{\mathrm{F}\}$ respectively, the program then solves for $\{U\}$, where $\{U\}$ is the displacement vector, which, like $\{F\}$, has some of its components known while others are unknown. The program first uses the Payne and Irons technique to separate the knowns from the unknowns in the stiffness equation $\{F\}=[K]\{U\}$ and then solves for $\{\mathrm{U}\}$ by back substitution and Gauss elimination BOWES and RUSSEL, [1], CLAUS-JURGEN BATH, [2].

Knowing $\{U\}$, the program proceeds to equation, $x x i$, and calculates vector $\{F\}$. At this stage, all the nodal forces and displacements are known. Program then goes back to each main material element and by using equation, viii, it calculates and prints horizontal stress $S_{x x}$ vertical stress $S_{y y}$ and shear stress $S_{x y}$. The program as well calculates and prints the major and minor principal stresses $\mathrm{P}_{1}$ and $\mathrm{P}_{2}$ respectively and the angle of inclination of the plane on which major and minor axis occur, $\theta$.

The program then proceeds to the second material (reinforcement) elements and calculates their axial stressesand prints them out. Having finished one case the program either terminates the execution, which is done by reading NEXT $=0$, or proceeds to another case by reading NEXT $=1$ in the data file. The sign convention followed in this program is the usual convention, identical to the plane coordinates sign for forces and displacements while that of stress is positive for tension and negative for compression. Since the program is designed to solve for in-plane forces and thermal strains, any deformations other than thermal have to be converted to an equivalent rise or fall in temperature in the input data, MOHAMED, [6].

\subsection{Flowchart of the Computer Program}

1

\section{Print title}

Read and Print Case Titles

Read Nodes Coordinates

Read and Write Main Material Data 
Read and Write Reinforcing Material Properties

Read Reinforcement Elements Data

Fill in $[S],\{\mathrm{U}\}$, and $\{\mathrm{F}\}$ with Zeros

Fill in [D] and [B] Matrices

Fill in A Matrix

Find $\mathrm{A}^{-1}$ and Write on 1

Number of Main Material Elements go to 33

Calculate Main Material Element Stiffness Matrix [E] and Thermal Force Vector FTE and add to $[\mathrm{S}]$ and $\{\mathrm{F}\}$

Calculate Work Equivalent Vector for Elements Own Weight and add to $\{\mathrm{F}\}$

Number of Reinforcing Elements $=0$ go to 66

Find Reinforcement Element Stiffness Matrix and add to [S]

Find Reinforcement Element Thermal Force vector and add to $\{\mathrm{F}\}$

66

Write [S] on 4

Read Known Forces and add to $\{\mathrm{F}\}$

Read Known Displacements and add to $\{\mathrm{U}\}$

Solve for $\{\mathrm{U}\}$ by method of Payne and Irons, Gauess Elimination

Read [S] from 4

Solve $\{\mathrm{F}\}=[\mathrm{S}] .\{\mathrm{U}\}$ print $\mathrm{F}$ and $\mathrm{U}$

Read $\mathrm{A}^{-1}$ from 1

For all Main Material Elements find and Print the 3 Plane Stresses Major and Minor Principle Stresses and Their Direction

Number of Reinforcing Elements $=0$ Go to 99

99

For all Reinforcing Elements Find and Print Axial Stresses

Read NEXT

NEXT: 0

Call EXIT

Go To 1

\section{Discussionsand Conclusions}

- Simple but efficient model is proposed for plane linear stress analysis using two elements for combined materials.

. Stiffness method is formulated for both elements and materials.

. Comprehensive Fortran V computer program for analysis is introduced.

- Despite vast availability of commercial programs that can perform any stress analysis, still young engineers need to comprehend methodology and theoretical aspects of the technique.

\section{References}

[1]. BOWES, W, H and RUSSEL, L. T, "Stress analysis by Finite Element for Practicing Engineers", 1975, D. C. Heath and Company, Lexington, Massachusetts, US

[2]. KLAUS-JURGEN BATHE, "Finite Element Procedures in Engineering Analysis" 1982, Prentice-Hall, Inc., Englewood Cliffs, New Jersey.

[3]. ROBERT D. COOK, "Concepts and Application of Finite Element Analysis", 1981, John Wiley \& Sons. Inc. Canada.

[4]. O. C. ZIENKIEWICZ "The Finite Element Method", $3^{\text {rd }}$ Edition 1977, McGraw-Hill, UK Limited.

[5]. I. M. SMITH and D. V. GRIFFITHS, "Finite Element Method" 2004, John Wiley \& Sons Itd. UK.

[6]. MOHAMED A. MOHAMED "Analysis of Low Rise Masonry Walls Subjected to Thermal and Moisture Deformations", 1984, Masters of Engineering Thesis Work, University Of Carlton, Ottawa, Canada. 